\title{
Kaczmarz Holography: Holographic Projection in the Fraunhofer Region
}

\author{
Miguel de V. El Guendy ${ }^{\mathrm{a}, \mathrm{b},{ }^{*}}$, Ralf Mouthaan ${ }^{\mathrm{a}}$, Benjamin Wetherfield ${ }^{\mathrm{a}}$, Andrew \\ Kadis $^{\text {a }}$, Peter J. Christopher ${ }^{a}$, Andrzej Kaczorowski ${ }^{b}$, Timothy D. Wilkinson ${ }^{\text {a }}$ \\ ${ }^{a}$ Centre of Molecular Materials, Photonics and Electronics, University of Cambridge, UK \\ ${ }^{b}$ VividQ Ltd., Research Division, Cambridge, UK
}

\begin{abstract}
The Kaczmarz algorithm is an iterative method for solving linear equations in the form of $A x=b$. It is widely used in computed tomography (CT) and digital signal processing (DSP) but has yet to be adopted in computer-generated holography (CGH). Phase retrieval algorithms such as GerchbergSaxton or Fienup are significantly more popular in this field, however, in this paper we propose a unique and alternative approach to projecting a replay field through Discrete Fourier Transform (DFT) matrices and have shown that there are legitimate benefits to implementing this approach. The gradient descent iteration mechanism adopted by Kaczmarz, for instance, provides finer granularity control over the individual pixels in the replay field. We consequently demonstrate the quality of the image is significantly improved when compared to Gerchberg-Saxton.
\end{abstract}

Keywords: Computer-Generated Holography, Kaczmarz, Gerchberg-Saxton.

\section{Introduction}

Photography by wavefront reconstruction, later known as holography has seen significant developments since its first publication in 1948 by Dennis Gabor. ${ }^{1}$ It works by recording onto a photographic plate, the interference pattern between a coherent reference light source and this same light source scattered off an object. A replay field is then projected by shining a coherent light source through this interference pattern.

With the advancement in computer technology, it is now possible to generate these interference patterns computationally without the need for photographic plate or physical object. The rapid availability of computer-controlled spatial light modulators (SLMs) facilitated the uptake of computer-generated holography for a wide range of applications from optical tweezing ${ }^{2}$ to telecommunications. ${ }^{3}$ SLMs modulate the spatial profile of an incoming coherent beam replacing the use of plates of conventional holography. The configuration of a hologram in modern computer-generated holography is dynamic, allowing for the projection of moving images as opposed to still images in conventional holography.

The development of these technologies has contributed towards the use of SLMs in augmented and virtual reality (AR and VR) systems. ${ }^{4-7}$ SLMs are capable of projecting three-dimensional images with appropriate depth cues that do not exhibit what is known as vergence-accommodation conflict (VAC). ${ }^{8-10}$ 
This paper focuses on projecting two-dimensional holographic images in the Fraunhofer region, also known as the far-field or replay field. In these systems, an SLM is configured to display a hologram that will modulate the coherent light beam displaying the desired image in the replay field. ${ }^{11-13}$ Goodman ${ }^{14}$ demonstrates that taking the discrete Fourier transform (DFT) of the aperture function of the SLM will reproduce the pattern of the image in the far-field. The SLM is a pixelated device in the diffraction field $(\mathrm{H})$ and as such is represented by a grid of discrete sampling points. The resultant replay field (R) is therefore also represented by discrete pixels and the relationship between both is shown in Fig 1. The Fourier transform is represented here as a DFT sum, however, it is usually represented as an integral. ${ }^{15}$

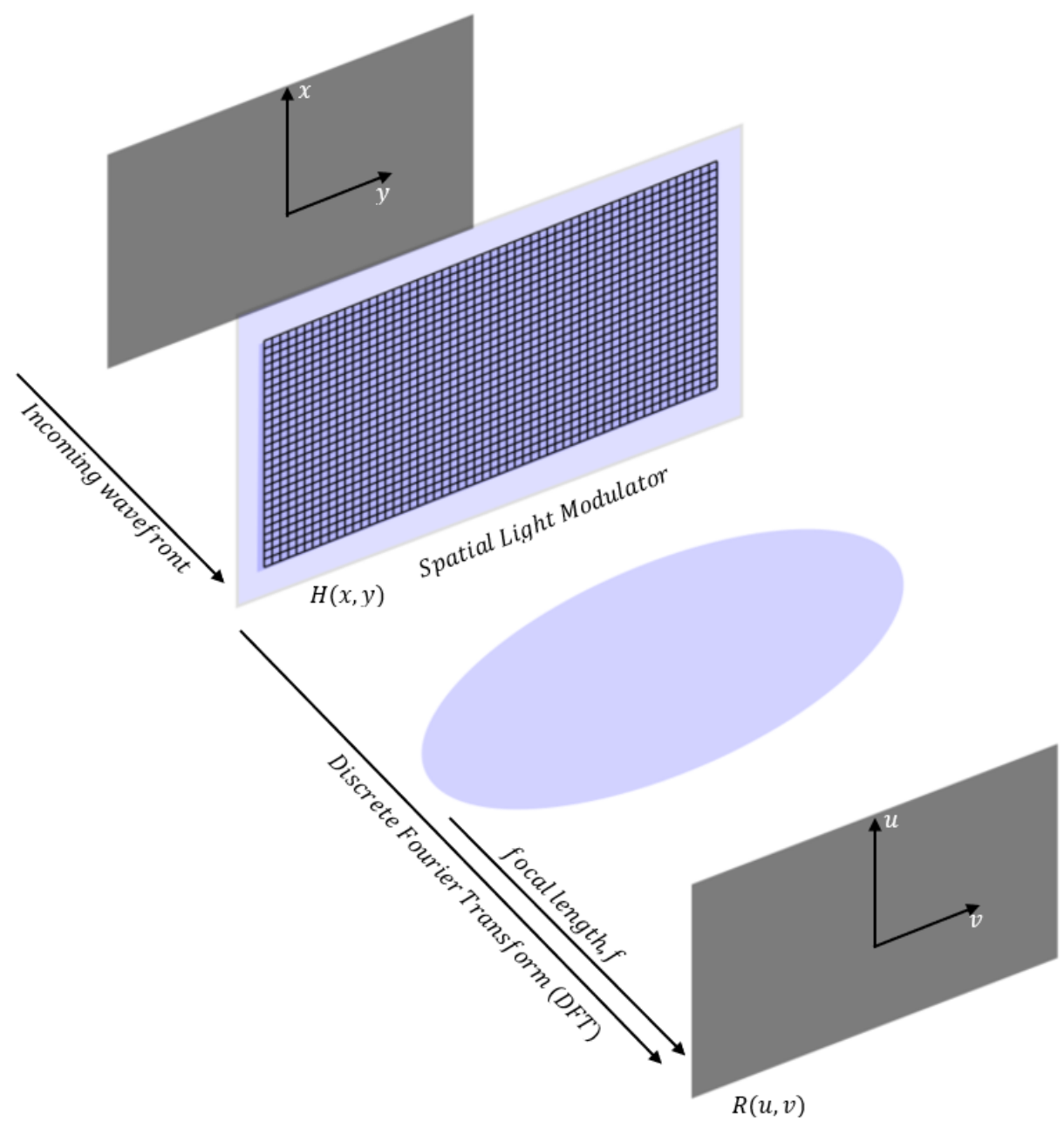

Fig 1: Diffraction/Hologram $(H)$ and replay $(R)$ fields coordinate system 
The diffraction field coordinates are indicated as $x$ and $y$ while replay field coordinates are portrayed as $u$ and $v$. It is worth noting, $N_{x}$ and $N_{y}$ represent the sizes of the diffraction and replay fields and are represented as follows.

$$
\begin{aligned}
& R_{u, v}=\mathcal{F}\left\{H_{x, y}\right\}=\frac{1}{\sqrt{N_{x} N_{y}}} \sum_{x=0}^{N_{x}-1} \sum_{y=0}^{N_{y}-1} H_{x, y} e^{-2 \pi i\left(\frac{u x}{N_{x}}+\frac{v y}{N_{y}}\right)} \\
& H_{x, y}=\mathcal{F}^{-1}\left\{R_{u, v}\right\}=\frac{1}{\sqrt{N_{x} N_{y}}} \sum_{u=0}^{N_{x}-1} \sum_{v=0}^{N_{y}-1} R_{u, v} e^{2 \pi i\left(\frac{u x}{N_{x}}+\frac{v y}{N_{y}}\right)}
\end{aligned}
$$

The SLM is restricted to modulating either the phase or the amplitude of an incident coherent light beam, not both. As a result, the challenge lies in constructing a hologram that can be displayed on this SLM under these restrictions and accurately projecting the target image in the replay field.

This paper analyzes the performance of the Kaczmarz algorithm in two-dimensional holographic image projection. Alternative methods such as Gerchberg-Saxton, ${ }^{16}$ Fienup ${ }^{17}$ and Wirtinger ${ }^{18}$ are very popular in this field and the aim of this paper is to introduce an alternative method for projecting these images. This method iterates pixel by pixel in the replay field and therefore offers finer granularity over theseindividual pixels, a property that distinguishes it from other algorithms. To facilitate comparison between the different algorithms, error metrics are applied to quantitively assess the difference between the replay field, $R$, and original target image, $T$. Mean squared error (MSE) and peak signal to noise ratio (PSNR) will be used throughout this investigation.

$$
\begin{gathered}
M S E(T, R)=\frac{1}{N_{x} N_{y}} \sum_{u=0}^{N_{x}-1} \sum_{v=0}^{N_{y}-1}\left|T_{u, v}-R_{u, v}\right|^{2} \\
P S N R(T, R)=20 \log _{10}\left(\frac{N_{x} N_{y}}{\sqrt{M S E}}\right)
\end{gathered}
$$

\section{Kaczmarz Algorithm}

First proposed by Polish mathematician Stefan Kaczmarz, ${ }^{19}$ this iterative algorithm is used in computational tomography (CT), digital signal processing (DSP), microscopy and metasurface antennas ${ }^{20-24}$ to solve linear equation systems in the form of $A x=b$. Typically, holography has been proven to work using Gerchberg-Saxton and Fienup algorithms since these are very effective in retrieving the phase of an image. However, the work carried out by $\mathrm{Wei}^{25}$ and subsequently $\mathrm{Tan}^{26}$ on the Kaczmarz algorithm suggests it is also very effective in solving this phase retrieval problem. 
This paper analyses the effectiveness of this algorithm in holography as well as its necessary adaptation to make it applicable to this use-case.

\subsection{Simple Kaczmarz Algorithm}

The Kaczmarz algorithm was originally devised to solve overdetermined linear systems of the form $A x=b .^{27}$ For $A_{r}$ the $r$ th row of $A$, and $A_{r}^{*}$ its conjugate transpose, the following update rule is used.

$$
x_{l+1}=x_{l}+\frac{b_{r}-A_{r} x_{l}}{\left\|A_{r}\right\|_{2}^{2}} A_{r}^{*}
$$

The row index $r$ is chosen sequentially with $r=l+1 \bmod m$.

Wei adapts the algorithm for the case where equality of magnitudes $|A x|=b$ is desired and phase is a free parameter (the generalised phase retrieval problem, ${ }^{25}$ ). The $l$ th phase term is determined as $\theta_{l}=\angle A_{r} x_{l}$, after which the original update rule has a natural extension.

$$
x_{l+1}=x_{l}+\frac{b_{r} e^{j \theta_{l}}-A_{r} x_{l}}{\left\|A_{r}\right\|_{2}^{2}} A_{r}^{*}
$$

Each iteration can be thought of as projecting the previous solution $x_{l}$ onto the hyperplane $H_{r}$ defined as follows.

$$
H_{r}^{\theta}=\left\{x: A_{r} x=b_{r} e^{i \theta}\right\} \text { where } \theta=\angle A_{r} x_{l}
$$

The above choice of $\theta$ is optimal in the sense that the distance from $x_{l}$ to $H_{r}^{\theta}$ is minimised for $\theta=\angle A_{R} x_{l}$.

\subsection{Randomized Kaczmarz Algorithm}

The randomized Kaczmarz algorithm was developed more recently by Strohmer and Vershynin, ${ }^{20}$ as they demonstrate an exponential convergence for a randomized version of the simple Kaczmarz method described in the previous section. This algorithm is shown to outperform the simple Kaczmarz method in terms of convergence rates, converging to a minimum faster than the simple Kaczmarz method. ${ }^{28}$ Wei derives further analytic convergence results for the analogous phase retrieval use case. ${ }^{25}$

This proposed version of the algorithm iterates through rows of $A$ randomly rather than sequentially. The probability of choosing each row in matrix $\mathrm{A}$ is proportional to the square of its Euclidean norm.

$$
P\{r=k\} \propto\left\|A_{k}\right\|_{2}^{2} \quad k=1,2, \ldots, m
$$




\section{Holography using Kaczmarz algorithm}

The Kaczmarz algorithm has been shown to be effective in multiple applications such as computer tomography, digital signal processing, microscopy as well as metasurface antennas. In this paper, however, we demonstrate it can also be effectively used in twodimensional holographic projections in the Fraunhofer region. Wei ${ }^{25}$ demonstrated this algorithm possessed phase retrieving properties as well as the possibility of converging better than other more established algorithms such as Gerchberg-Saxton and Wirtinger flow. Nevertheless, this algorithm has to be adapted to suit our problem of projecting a holographic image in the far-field region.

Rather than using Fast Fourier Transforms (FFTs), as in Gerchberg-Saxton, Kaczmarz phase retrieval algorithm uses DFT matrices to iteratively recover the original target image. Multiplying a two-dimensional DFT matrix with the hologram will reproduce a two-dimensional replay field in the far-field.

For this linear system algorithm to work, $A$ is the DFT matrix, $x$ is the image hologram and $b$ is the target image. Both $x$ and $b$ are vectors. Much like Gerchberg-Saxton, the algorithm starts with a random hologram and the product of the DFT matrix and the hologram is consistently compared with the target image $b$. DFT matrices usually refer to the 1D DFT matrix, however, we had to create a 2D DFT matrix for this algorithm to work accordingly. The expression below demonstrates how the linear system was adapted to implement holography.

$$
\left(\begin{array}{ccc}
a_{11} & \ldots & a_{1 n} \\
\vdots & \ddots & \vdots \\
a_{m 1} & \ldots & a_{m n}
\end{array}\right)\left(\begin{array}{c}
x_{11} \\
\vdots \\
x_{m 1}
\end{array}\right)=\left(\begin{array}{c}
b_{11} \\
\vdots \\
b_{m 1}
\end{array}\right)
$$

\section{D DFT Holo Target}

One of the challenges faced in this project was constraining the amplitude of the hologram. While the replay field is naturally constrained, it was found that this was not the case in the diffraction plane. As previously mentioned, the SLM has certain restrictions and in order to successfully project an image in the replay field, one must construct a hologram under these constraints. One of these constraints is the amplitude of the hologram must be equal to one.

\section{Kaczmarz Convergence of Holographic Images}

The following test images of $128 \times 128$ pixels, seen in Fig 2, were used throughout this investigation. The performance of Kaczmarz in projecting a replay field in the far-field was compared to Gerchberg-Saxton. The Wirtinger algorithm, expressed by $\mathrm{Wei}^{25}$ is optimized for images in near-field applications ${ }^{18,29}$ and this is not compared here as this 

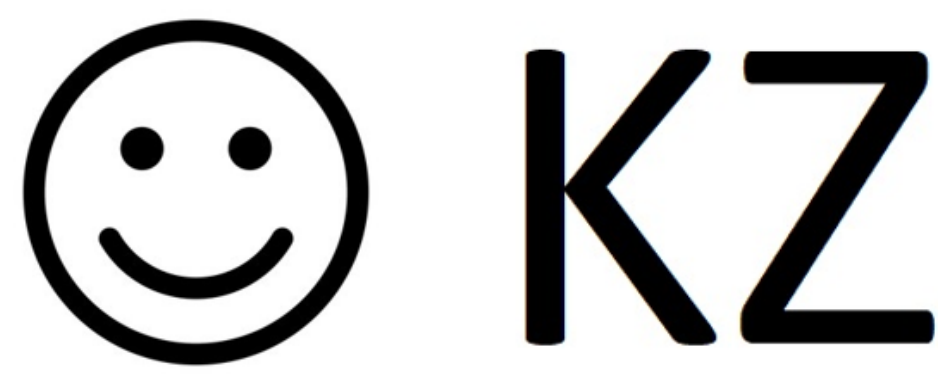

Fig 2: Target Images used throughout this investigation

represents a different optical configuration where the image is formed before the far-field. Each algorithm was run for ten iterations and their respective image reconstructions were quantitatively analysed and compared.

\subsection{Simple Kaczmarz}

The first variant of the algorithm presented is the simple Kaczmarz algorithm. Fig 3 displays the performance of Kaczmarz and Gerchberg-Saxton after 10 image iterations. One iteration of Kaczmarz is counted after all image rows have been processed whereas Gerchberg-Saxton performs the fast Fourier transform of one image all at once. Whilst Gerchberg-Saxton utilises a more computationally-efficient mechanism, Kaczmarz offers an interesting approach to projecting in the far-field and converges to a minimum in fewer iterations when compared to GS, as seen in Fig 4.

\subsection{Randomized Kaczmarz}

The randomized version of this algorithm is also compared to Gerchberg-Saxton and while it is similarly converging faster after 10 iterations, the image quality looks poorer than the simple Kaczmarz version presented earlier. This is illustrated in Fig 5. Much like simple Kaczmarz, the error metrics in Fig 6 show randomized Kaczmarz converges in fewer iterations when compared to Gerchberg-Saxton.

\section{Experimental Results}

This section compares the simple Kaczmarz algorithm to Gerchberg-Saxton on a holographic projector. Much like the previous section, the image quality of 10 iterations of Kaczmarz will be compared to 10 iterations of Gerchberg-Saxton. It is worth noting the comparison between both will be made using a binary SLM as opposed to the multi-phase approach adopted in the previous section. Therefore, rather than comparing these results to simulations we compared them with respect to each other under the exact same conditions. 

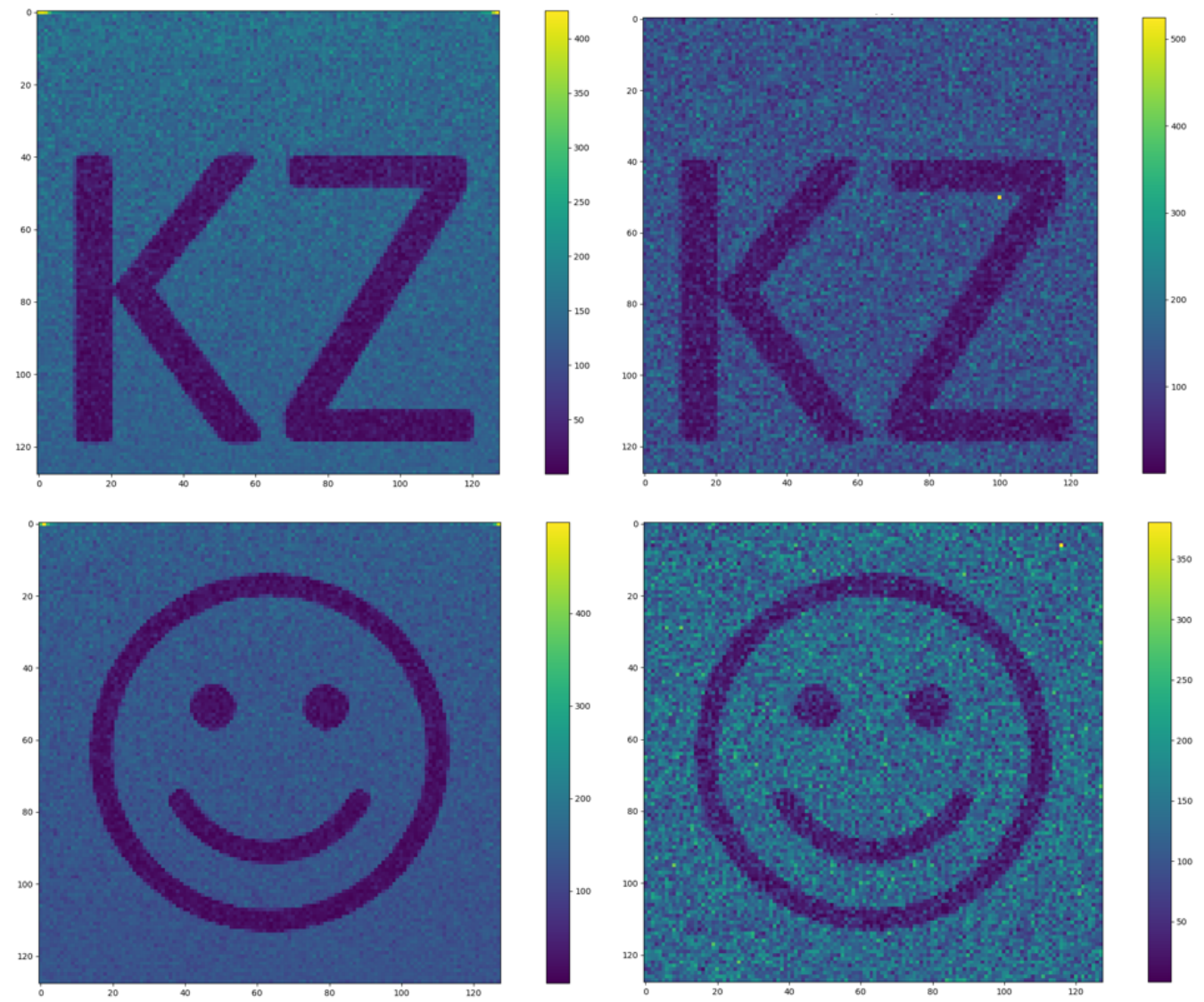

Fig 3: Images retrieved through Simple Kaczmarz (Left) and Gerchberg-Saxton (Right)
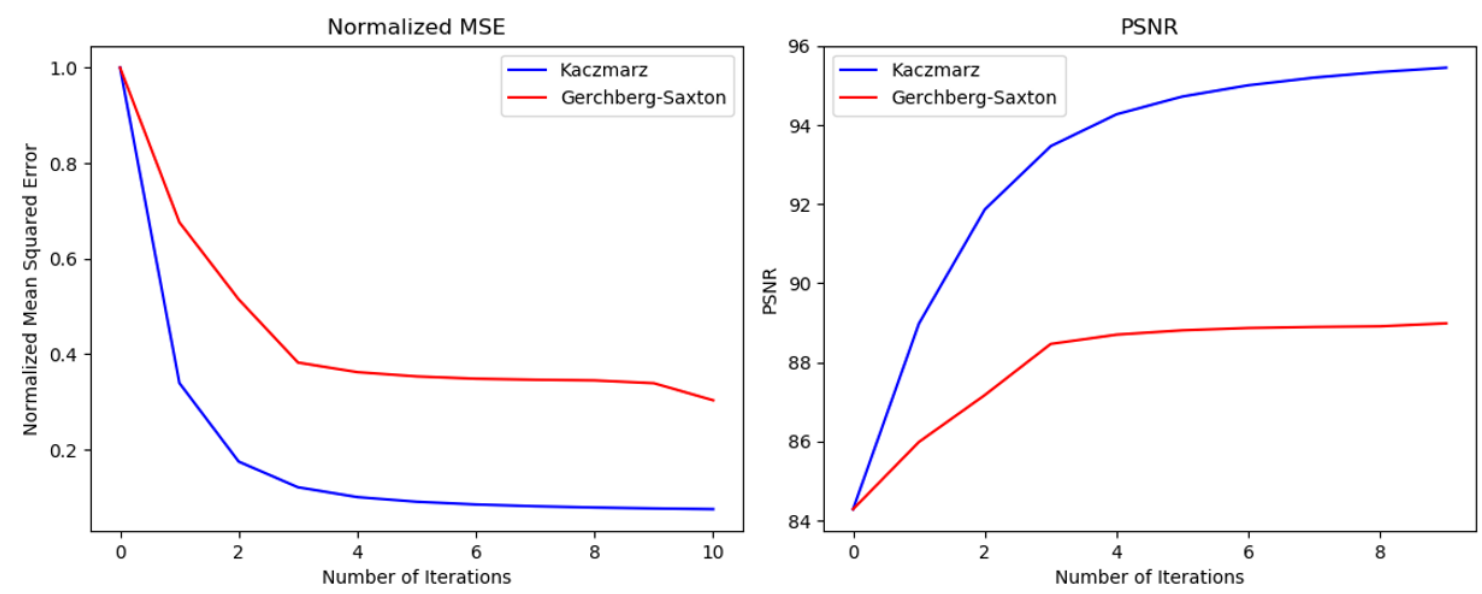

Fig 4: Error Metrics of Simple Kaczmarz and Gerchberg-Saxton 

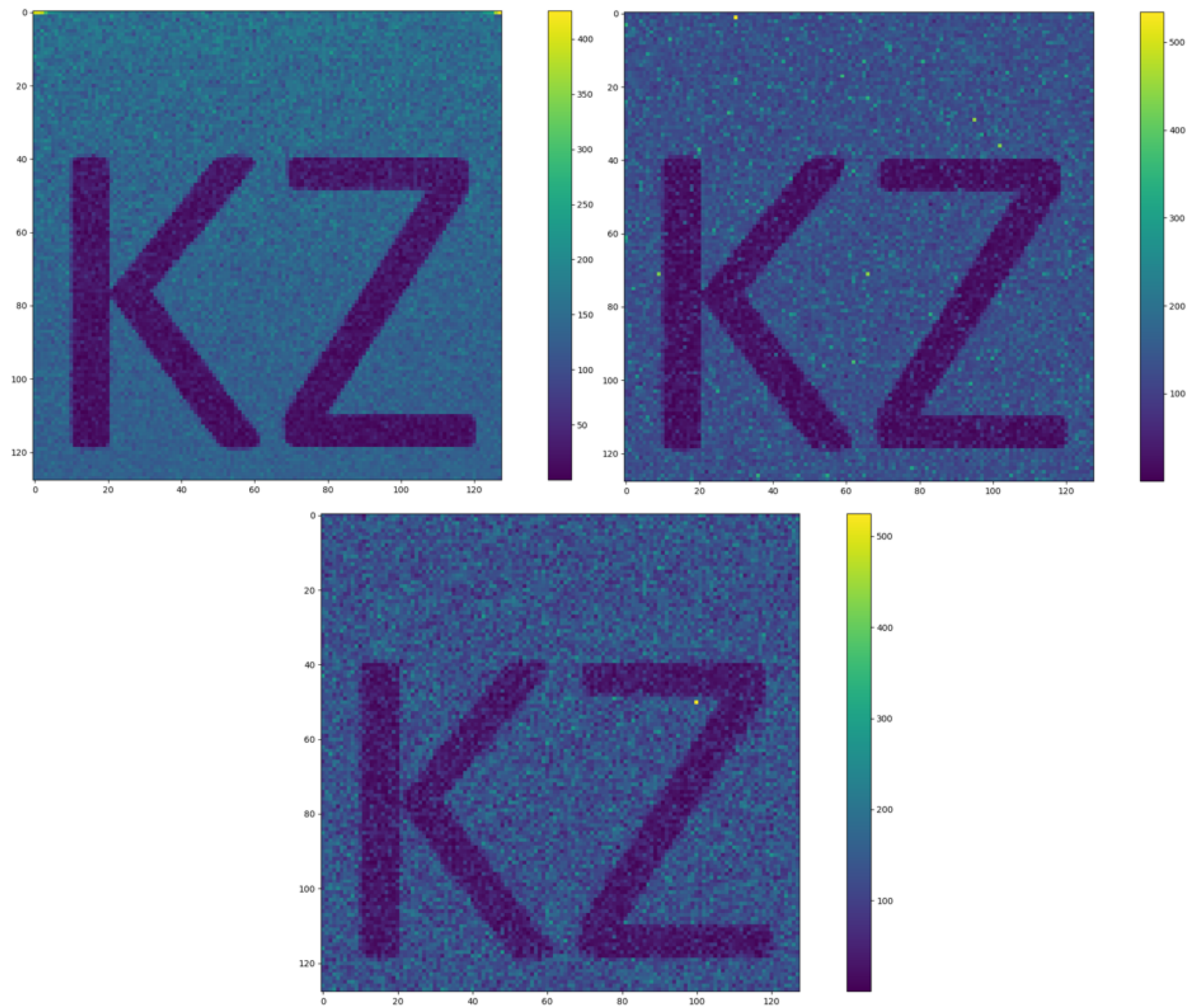

Fig 5: Images retrieved through Simple Kaczmarz (Top Left), Randomized Kaczmarz (Top Right) and Gerchberg-Saxton (Bottom) 

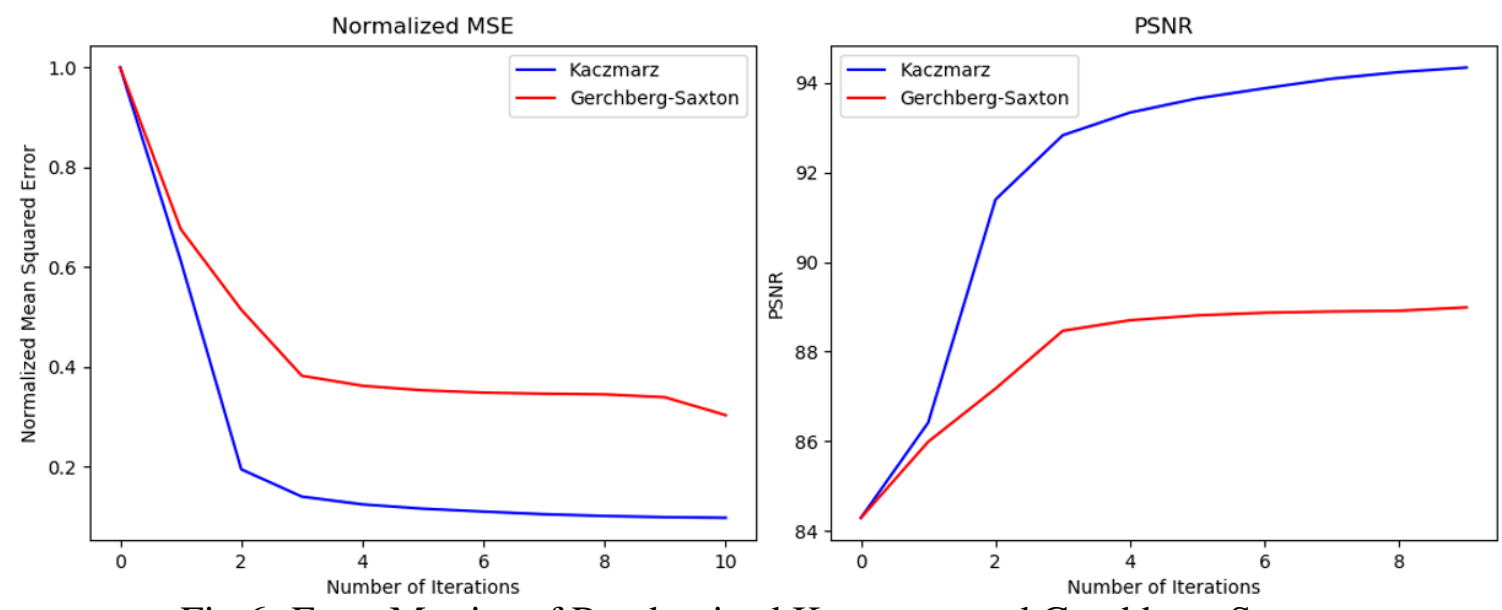

Fig 6: Error Metrics of Randomized Kaczmarz and Gerchberg-Saxton

A picture of the projector and its setup can be seen in Fig 7. A $532 \mathrm{~nm}$ laser is expanded by lens L1 (focal length $5 \mathrm{~mm}$ ) and then collimated by L2 (focal length $150 \mathrm{~mm}$ ) before reaching the SLM. The beam is then subsequently scaled down by lens L2 and polarized after passing the beam-splitter. It is then collimated by lens $\mathrm{L} 3$ (focal length $3.4 \mathrm{~mm}$ ) before being projected on a plane surface ahead of the projector. The SLM used is a 4DD SXGA ferroelectric LC device with 1280x1024 pixels.

Fig 8 demonstrates the results obtained. It is noticeable that, much like what was verified in the previous section, the simple Kaczmarz mechanism displays a higher quality image when compared to Gerchberg-Saxton after 10 iterations.

\section{Future Work}

This investigation presents a new mechanism to projecting a holographic image in the far-field. Whilst it is comparatively slower than algorithms like Gerchberg-Saxton, the improved image quality seen in the results above could be used if, for instance, a section of a larger image would require quality enhancement.

Arguably, whilst using matrices and vectors to project these replay field leads to improved image quality, it also leads to very large memory usage. The multiple matrix computations are both computationally and memory intensive, especially when compared to Gerchberg-Saxton. For images that require that extra quality, however, it becomes a legitimate option.

We have analysed its performance on image quality but there are other metrics that are an equally interesting avenue to pursue. These include, for instance, assessing the effects on speckle noise and camera shot noise. Iterating on a pixel by pixel basis in the replay 


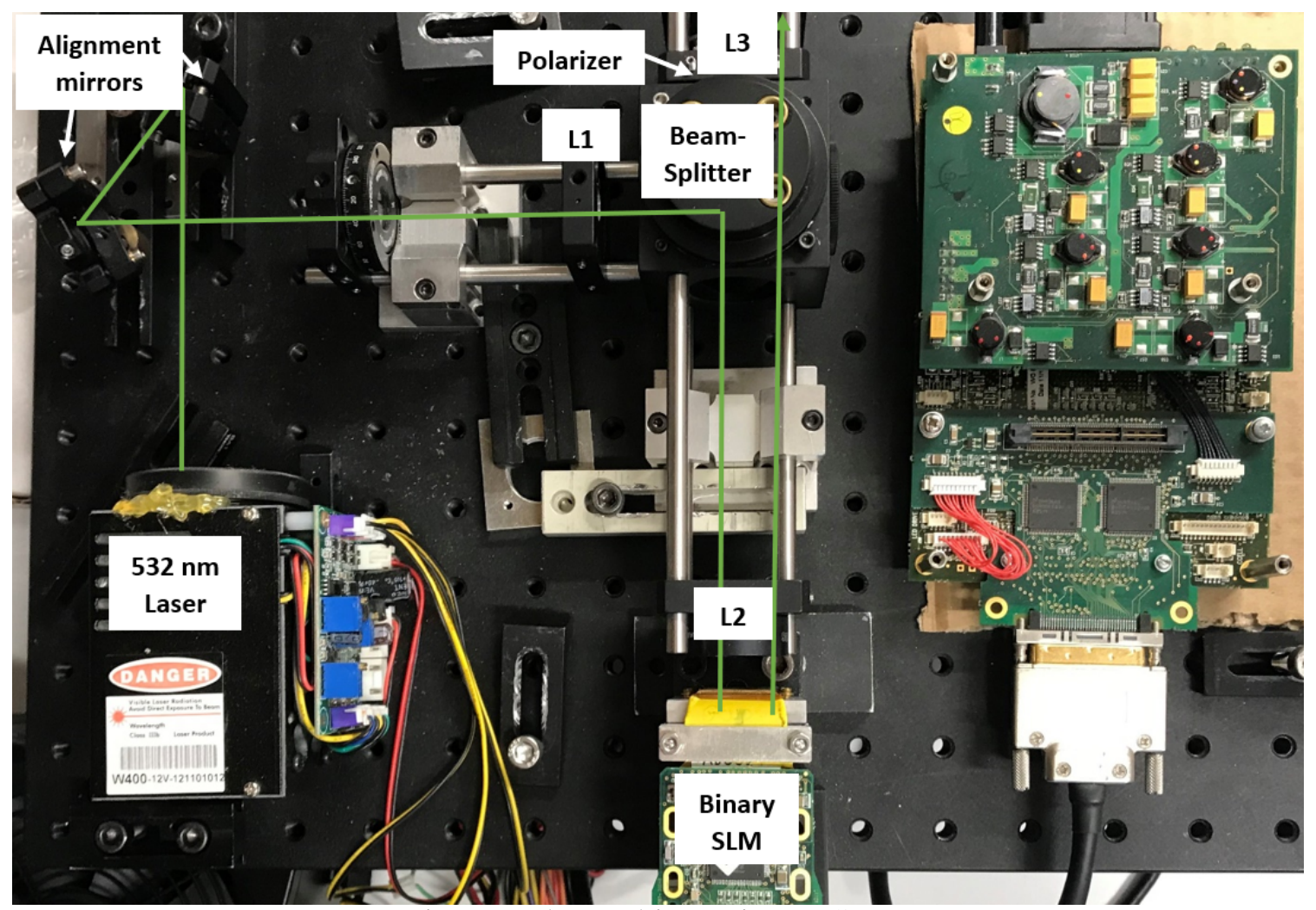

Fig 7: Holographic Projector Setup

field gives the user finer granularity control over each pixel. We have seen significant improvements in image quality but we need to analyse the effects on noise further.

\section{Conclusions}

This method provides an alternative to using FFTs by doing a series of deterministic matrix computations. FFTs determines all pixels of whole images at once while Kaczmarz iterates row by row in the DFT matrix and pixel by pixel in the replay field. However, while it is comparatively slower than Gerchberg-Saxton, it can be seen from Figures 3 and 5 that the image quality is significantly improved after the same number of iterations.

After simulating the effects of using Kaczmarz in holography we have successfully implemented the simple version of this algorithm on a holographic projector with binary SLM and we have shown the simple Kaczmarz demonstrates a higher image quality when compared to Gerchberg-Saxton after 10 iterations. This is due to the finer granularity control over individual pixels. 

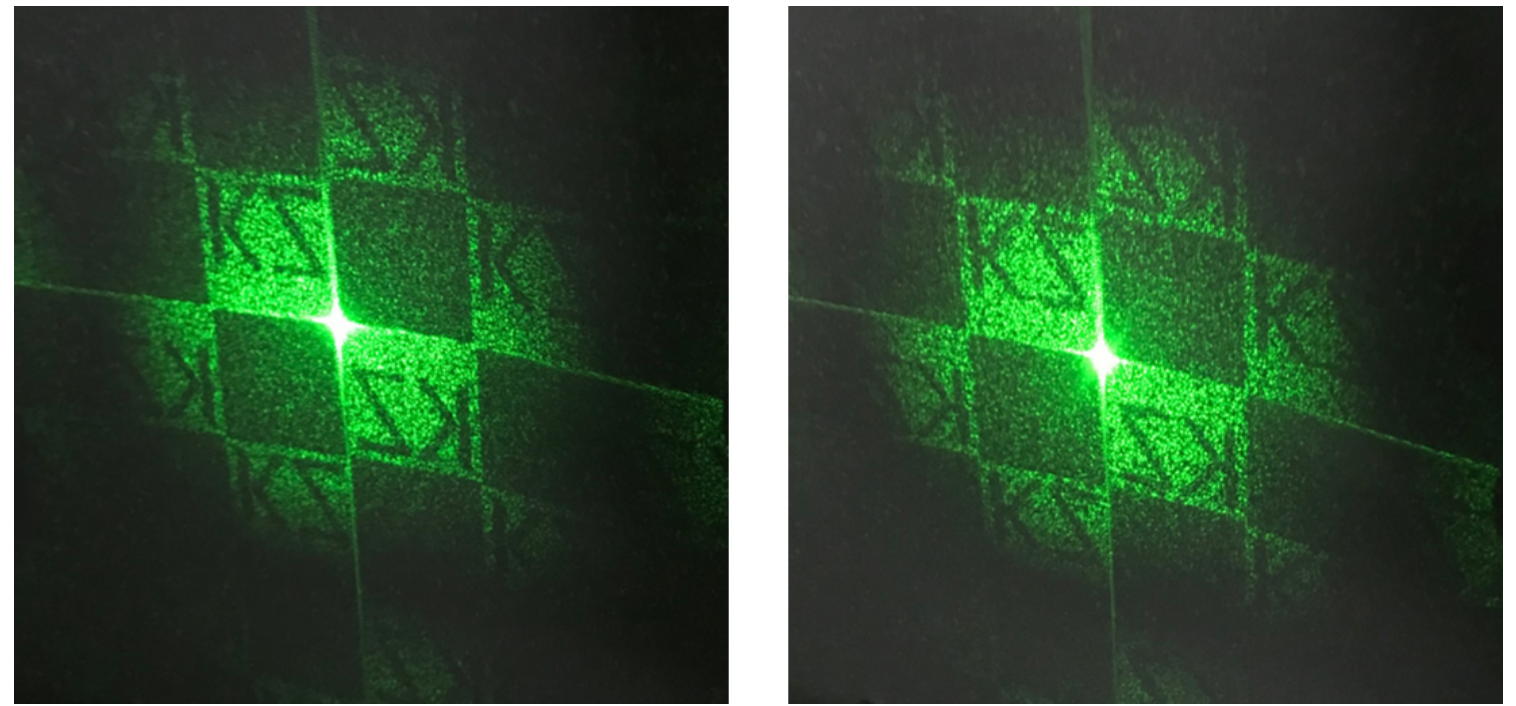

Fig 8: Kaczmarz (left) and Gerchberg-Saxton (right) replay fields projected from a binary SLM. MSE for images are 105.4 and 109.5 for Kaczmarz and Gerchberg-Saxton respectively. The PSNR is 55.9 and 55.7 for Kaczmarz and Gerchberg-Saxton respectively.

While there is still work to optimize this mechanism further, this investigation shows the Kaczmarz algorithm can be successfully implemented in holography and results demonstrate higher quality replay fields compared to Gerchberg-Saxton.

\section{Funding}

The authors would like to thank the Engineering and Physical Sciences Research Council (EP/L015455/1 and EP/L016567/1), VividQ Ltd and Cambridge Trust for financial support during the period of this research.

\section{Disclosures}

The authors declare no conflicts of interest.

\section{References}

1 D. Gabor, "A new microscopic principle," Nature , 1948.

2 J. Grieve, A. Ulcinas, S. Subramanian, G. Gibson, M. Padgett, D. Carberry, and M. Miles, "Hands-on with optical tweezers: a multitouch interface for holographic optical trapping," Optics Express 17(5), pp. 3595-3602, 2009.

3 W. Crossland, T. Wilkinson, I. Manolis, M. Redmond, and A. Davey, "Telecommunications applications of lcos devices," Molecular Crystals and Liquid Crystals 375(1), pp. 1-13, 2002. 
4 T. Widjanarko, M. El Guendy, A. Spiess, D. Sullivan, T. Durrant, O. Tastemur, A. Newman, D. Milne, and A. Kaczorowski, "Clearing key barriers to mass adoption of augmented reality with computer-generated holography," in Optical Architectures for Displays and Sensing in Augmented, Virtual, and Mixed Reality (AR, VR, MR), 11310, p. 113100B, International Society for Optics and Photonics, 2020.

5 Z. He, X. Sui, G. Jin, and L. Cao, "Progress in virtual reality and augmented reality based on holographic display," Applied optics 58(5), pp. A74-A81, 2019.

6 A. Maimone, A. Georgiou, and J. S. Kollin, "Holographic near-eye displays for virtual and augmented reality," ACM Transactions on Graphics (Tog) 36(4), pp. 1-16, 2017.

7 L. Shi, B. Li, C. Kim, P. Kellnhofer, and W. Matusik, “Towards real-time photorealistic 3d holography with deep neural networks," Nature 591(7849), pp. 234-239, 2021.

8 T. Shibata, J. Kim, D. M. Hoffman, and M. S. Banks, "Visual discomfort with stereo displays: effects of viewing distance and direction of vergence-accommodation conflict," in Stereoscopic Displays and Applications XXII, 7863, p. 78630P, International Society for Optics and Photonics, 2011.

9 K. Ukai and P. A. Howarth, "Visual fatigue caused by viewing stereoscopic motion images: Background, theories, and observations," Displays 29(2), pp. 106-116, 2008.

10 T. Shibata, J. Kim, D. M. Hoffman, and M. S. Banks, "The zone of comfort: Predicting visual discomfort with stereo displays," Journal of vision 11(8), pp. 11-11, 2011.

11 P. J. Christopher, R. Mouthaan, M. El Guendy, and T. D. Wilkinson, "Linear-time algorithm for phase-sensitive holography," Optical Engineering 59(8), p. 085104, 2020.

12 P. J. Christopher, R. Mouthaan, V. Bheemireddy, and T. D. Wilkinson, "Improving performance of single-pass real-time holographic projection," Optics Communications 457, p. 124666, 2020.

13 P. J. Christopher, J. D. Lake, D. Dong, H. J. Joyce, and T. D. Wilkinson, "Improving holographic search algorithms using sorted pixel selection," JOSA A 36(9), pp. 14561462, 2019.

14 J. W. Goodman, Introduction to Fourier optics, Roberts and Company Publishers, 2005.

15 W. T. Cochran, J. W. Cooley, D. L. Favin, H. D. Helms, R. A. Kaenel, W. W. Lang, G. C. Maling, D. E. Nelson, C. M. Rader, and P. D. Welch, "What is the fast fourier transform?," Proceedings of the IEEE 55(10), pp. 1664-1674, 1967. 
16 R. W. Gerchberg, "A practical algorithm for the determination of phase from image and diffraction plane pictures," Optik 35, pp. 237-246, 1972.

17 J. R. Fienup, "Phase retrieval algorithms: a comparison," Applied optics 21(15), pp. 2758-2769, 1982.

18 P. Chakravarthula, Y. Peng, J. Kollin, H. Fuchs, and F. Heide, "Wirtinger holography for near-eye displays," ACM Transactions on Graphics (TOG) 38(6), pp. 1-13, 2019.

19 S. Karczmarz, "Angenaherte auflosung von systemen linearer glei-chungen," Bull. Int. Acad. Pol. Sic. Let., Cl. Sci. Math. Nat. , pp. 355-357, 1937.

20 T. Strohmer and R. Vershynin, "A randomized kaczmarz algorithm with exponential convergence," Journal of Fourier Analysis and Applications 15(2), pp. 262-278, 2009.

21 Y. Jiao, B. Jin, and X. Lu, "Preasymptotic convergence of randomized kaczmarz method," Inverse Problems 33(12), p. 125012, 2017.

22 C. Byrne, "A unified treatment of some iterative algorithms in signal processing and image reconstruction," Inverse problems 20(1), p. 103, 2003.

23 C. Schretter, D. Blinder, S. Bettens, H. Ottevaere, and P. Schelkens, "Regularized non-convex image reconstruction in digital holographic microscopy," Opt. Express 25, pp. 16491-16508, Jul 2017.

24 Z. Luo, Y. Cheng, K. Cao, Y. Qin, and K. Liu, "A phase-calibrated computational imaging method based on programmable metasurface," in 2019 IEEE 2nd International Conference on Electronic Information and Communication Technology (ICEICT), pp. 778-780, 2019.

$25 \mathrm{~K}$. Wei, "Solving systems of phaseless equations via kaczmarz methods: A proof of concept study," Inverse Problems 31(12), p. 125008, 2015.

26 Y. S. Tan and R. Vershynin, "Phase retrieval via randomized kaczmarz: Theoretical guarantees," Information and Inference: A Journal of the IMA 8(1), pp. 97-123, 2019.

$27 \mathrm{X}$. Chen, "The kaczmarz algorithm, row action methods, and statistical learning algorithms," Frames and harmonic analysis 706, pp. 115-127, 2018.

28 Y. Jiang, G. Wu, and L. Jiang, "A kaczmarz method with simple random sampling for solving large linear systems," arXiv preprint arXiv:2011.14693 , 2020.

29 P. Chakravarthula, Y. Peng, J. Kollin, F. Heide, and H. Fuchs, "Computing high quality phase-only holograms for holographic displays," in Optical Architectures for Displays and Sensing in Augmented, Virtual, and Mixed Reality (AR, VR, MR), 11310, p. 1131006, International Society for Optics and Photonics, 2020. 
Miguel de V. El Guendy is currently pursuing a $\mathrm{PhD}$ in the department of Engineering at the University of Cambridge where he previously graduated with an M.Res. degree. Prior to this, he worked as a Senior Engineer at Qualcomm and holds an M.Eng. degree from Newcastle University.

Ralf Mouthaan pursued his undergraduate studies in Physics at the University of Nottingham. He subsequently spent eight years at the UK's National Physical Laboratory as a microwave metrologist before joining the University of Cambridge to obtain his $\mathrm{PhD}$. Ralf is now a Research Associate at the Cavendish Laboratory, with a research interest in holographic control of light propagation in optical waveguides.

Benjamin Wetherfield graduated summa cum laude (Highest Honors) from Harvard University in 2017 with a degree in Applied Mathematics. He is currently studying for a $\mathrm{PhD}$ at the University of Cambridge in the department of Electrical Engineering. His research interests are in fast approaches to computer-generated holography and optical-aided computation..

Andrew Kadis originally graduated from the University of Adelaide, Australia in 2010 with a first class degree in Engineering and a bachelor of Computer Science. Before commencing his $\mathrm{PhD}$ studies in 2018, he had considerable experience in industry; working on embedded systems in drones, medical devices and life sciences equipment. He is currently at the University of Cambridge working with Prof. Tim Wilkinson in the CMMPE group.

Peter J. Christopher is a Research Associate at the University of Cambridge with interests in all things holographic. He is also a Research Fellow at Emmanuel College and co-founder of Exobotics and ProSpectral. In the past he has been a successful entrepreneur, software engineer and civil engineer.

Andrzej Kaczorowski is the Co-Founder \& Chief Scientist of VividQ, a deep tech start-up with world-leading expertise in 3D holographic display. Andrzej holds an interdisciplinary scientific background. He earned a PhD and MRes in Photonic Systems Development from the University of Cambridge, and a BSc (Hons.) in Physics and Computer Science from King's College London.

Timothy D. Wilkinson is the Professor of Photonic Engineering at the University of Cambridge and a Fellow of Jesus College. He has had a long term research interest into the applications of holograms, optical information processing, liquid crystal (LC) devices, nanophotonics and related applications. 